\title{
Laboratorio de desarrollo en Educación Superior con alumnado de Altas Capacidades y/o Alto rendimiento Development Laboratory in Higher Education with High Ability and / or High performance students
}

\author{
Angélica González Arrieta ${ }^{1}$, María Belén Pérez Lancho ${ }^{1}$, José Rafael García-Bermejo-Ginner ${ }^{1}$, Iván Álvarez Navia ${ }^{1}$, \\ Ángel Luis Sánchez Lázaro ${ }^{1}$, Juan Andrés Hernández Simón ${ }^{1}$, Teresa Martín García ${ }^{2}$, Pastora Isabel Vega Cruz ${ }^{1}$ \\ email angelica@usal.es, email lancho@usal.es, email coti@usal.es, email inavia@usal.es, email alsl@usal.es, email \\ jahsimon@usal.es, email teresam@usal.es, email pvega@usal.es
}

\author{
${ }^{1}$ Informática y Automática \\ Universidad de Salamanca \\ Salamanca, España
}

\author{
${ }^{2}$ Sociología y Comunicación \\ Universidad de Salamanca \\ Salamanca, España
}

\begin{abstract}
Resumen- En el marco universitario, como en el resto del sistema educativo, buscamos alcanzar los niveles de excelencia adecuados para una óptima formación académica, así como para garantizar la capacitación profesional de los estudiantes. Centrándonos en respetar el ritmo de aprendizaje del estudiante, en concreto el del alumnado de altas capacidades y/o alto rendimiento, proponemos crear, en los grupos de investigación de excelencia, laboratorios de desarrollo para potenciar el aprendizaje de este tipo de alumnos. Solicitamos en este sentido el respaldo del Ministerio de Educación y Formación Profesional. Previamente tenemos que concienciar al profesorado universitario que este tipo de alumnos tiene necesidades educativas especiales, porque no siempre los de alumnos altas capacidades son también alumnos de alto rendimiento. Por esta razón, es preciso formar al profesor universitario en la detección de estos alumnos, capacitándole para que lleve a cabo la oportuna intervención. Finalmente, consideramos que es el propio alumno de altas capacidades quien, consciente de su especial situación, debe exigir sus derechos.
\end{abstract}

Palabras clave: Altas capacidades, alto rendimiento, innovar, talento, atención a la diversidad, intervención

Abstract- In the university framework, as in the rest of the educational
system, one tries to achieve the levels of excellence required for an
optimum academic training as well as the professional training of
students. We focus on respecting the student's learning pace,
specifically that of students with higher capabilities and / or higher
performance. Hence, we propose the creation of development labs in
research groups of excellence, which will enhance learning for these
groups of students. For this we require the help from the Ministry of
Education. Clearly, we must make university teachers aware that these
types of students have special educational needs. Further, students with
high abilities are not always high-achieving students. Therefore, we
must train the university teacher in the detection of these students, and
in proper intervention in those cases. Finally, students with high
capabilities must be made conscious of their need for a different
approach: they should learn to demand their rights, for the good of all.
Keywords: High abilities, high performance, innovate, talent, attention to diversity, intervention

\section{INTRODUCCIÓN}

La implantación de los nuevos planes de estudio de los grados universitarios, basados en una formación de competencias generales y específicas, ha exigido una renovación de las metodologías de enseñanza-aprendizaje y de los procesos de evaluación de la universidad. El estudiante se ha convertido en el sujeto central del proceso y la mejora de su aprendizaje es la finalidad de los procesos de innovación implementados. El profesorado se mantiene fiel a su compromiso de mejorar continuamente para alcanzar los niveles de excelencia necesarios para una adecuada formación académica y capacitación profesional de sus estudiantes. En este marco nos vamos a centrar en la atención a la diversidad, en concreto a los alumnos de altas capacidades (AACC) y los de alto rendimiento (AR).

Todas las teorías sobre altas capacidades, así como las técnicas y estrategias de evaluación e intervención, no serían útiles si no nos condujeran finalmente a tener en cuenta la individualidad de la persona con altas capacidades. Se trata de la propuesta educativa que presentamos para el alumnado universitario.

Son muchos los estudios llevados a cabo con alumnos de las primeras etapas de escolarización y en enseñanza media. Existen modelos de intervención internacionales, como el de Joseph S. Renzulli, (Renzulli \& Reis, 1997) y nacionales, como el de Ceferino Artiles, pioneros en practicar la mentoría como medida educativa eficaz para el desarrollo del talento (Fernández-Molina, 2021). Otro ejemplo es el desarrollado en el programa de mentorías universitarias para alumnado AACC de $1^{\circ}$ de ESO a Bachillerato y Ciclos Formativos, en el marco del proyecto MENTORAC de la universidad de Málaga (The mentorac project-UMA, s.f.). Ahora, ¿cómo intervenimos con 
alumnos universitarios de altas capacidades y/o alto rendimiento?

Muchos de los alumnos de altas capacidades sufren fracaso escolar en la etapa de secundaria y, a pesar de tener un perfil intelectual ideal para estudiar determinados grados, en ocasiones no consiguen acceder a ellos. Una de las razones es que, como su expediente académico no refleja su capacidad, no consiguen la nota mínima exigida para realizar esos estudios universitarios y nos preguntamos ¿por qué en la universidad hay plazas reservadas para deportistas de alto nivel y de alto rendimiento, para estudiantes con discapacidad, etc. (BOE, 2014) y no para personas con altas capacidades? Posiblemente la sociedad no reconoce sus necesidades especiales y por lo tanto tampoco en el mundo universitario.

Como equipo de trabajo dentro de una universidad ponemos de manifiesto que raro es el año que desde el servicio de asuntos sociales (SAS) no nos comuniquen que uno o dos alumnos por materia tienen una discapacidad de diferente tipo y que necesitan una atención especial (trastorno del espectro autista, discapacidad auditiva, síndrome de trastorno del aprendizaje, trastorno por déficit de atención e hiperactividad, etc.) pero nunca, en más de los 30 años que llevamos algunos como docentes universitarios nos ha llegado la necesidad de atender a una persona con altas capacidades. Como profesionales en la docencia nos preguntamos, ¿será que no necesitan atención especial?, ¿será que el alumno desconoce que en las universidades hay servicio de orientación universitario en el que el estudiante de altas capacidades tiene cabida?, ¿será que el profesor universitario desconoce que tiene que atender de forma diferente las necesidades de este tipo de estudiante?

Sin duda en las universidades tenemos que intervenir en los estudiantes que tienen necesidades específicas. Los estudiantes con mayor capacidad también las tienen, ya que muestran, por ejemplo, un mayor ritmo de aprendizaje. Para tener en cuenta este aspecto sería deseable permitirles adaptarse a un aprendizaje de mayor rapidez y profundidad de lo exigido. Esta elección ha de ser voluntaria, ya que estudiar con mayor intensidad que el resto no siempre tiene un lado productivo, ha de ser el estudiante quien escoja esta opción siendo consciente de que también va a ser evaluado con mayor rigor, puesto que verse obligado podría tener inconvenientes como la frustración (Tomlinson, 2013).

Por otra parte, es de vital importancia crear un ambiente agradable y enriquecedor para que el estudiante con altas capacidades no pierda el entusiasmo por aprender. Este ambiente ha de implantarse desde el primer indicio de curiosidad o interés por el estudio, el cual se ve muy bien proyectado en muchas materias universitarias.

Todas las teorías sobre altas capacidades, así como las técnicas y estrategias de evaluación e intervención, no serían útiles si no nos condujeran finalmente a respetar la individualidad de la persona con altas capacidades y/o alto rendimiento. Esta es la idea en la que se enmarca la propuesta educativa que presentamos para este tipo de alumnado universitario.

\section{CONTEXTO}

El concepto altas capacidades incluye dos realidades diferentes: el talento y la superdotación. Las personas con talento son aquellas que destacan de forma muy especial en uno o varios ámbitos o instrucciones diferenciados en los test de inteligencia tradicionales, pero no en todos. Los superdotados son los que tienen una capacidad de aprendizaje muy superior a la media en todos los ámbitos. Tanto unos como otros necesitan una educación diferenciada porque tienen capacidades de aprendizaje diferentes al resto de los alumnos.

Podemos reflexionar durante horas sobre las necesidades y el cuidado de los estudiantes con altas capacidades, pero ¿cómo son estos estudiantes y como es el proceso de identificación? Son muchos los escritos que definen a estas personas, sobre todo desde las características observables y el proceso de identificación, pero en determinados casos, estas definiciones vienen impuestas por la normativa educativa del territorio en el que se encuentren. Estas descripciones suelen ser poco concretas o muy ambiguas. Podemos tomar como ejemplo la definición vigente en Castilla y León, en esta descripción se puede observar un concepto más amplio y benévolo (Educacyl., 2021):

"Se entiende por alumnado con altas capacidades intelectuales aquel que presenta necesidades educativas derivadas de su alta capacidad intelectual, de la adquisición temprana de algunos aprendizajes o de sus habilidades especificas o creativas en determinadas áreas o materias y, por tanto, precisa de una respuesta educativa distinta y diferenciada respecto a otras necesidades especificas de apoyo educativo."

El proceso de identificación está asociado a un protocolo de evaluación psicopedagógica que realizan los profesionales del departamento de orientación de los centros educativos. Esta evaluación se llega a tergiversar incontables veces para adaptarlo a unos criterios y condiciones más rígidas y exigentes como, por ejemplo, el corte en pruebas psicométricas, características socioemocionales o calificaciones escolares. Estos criterios pueden llegar a ser negativos ya que deja a gran parte del alumnado candidato excluido de cualquier tipo de atención. Así es como las administraciones educativas reconocen a este tipo de personas, pero más allá de esos criterios, suelen considerarse otros factores como una exigencia de que el alumno muestre un comportamiento ejemplar, que tenga una alta implicación en la dinámica escolar, que sea creativo que hayan superado un determinado cociente intelectual en pruebas de inteligencia.

Realmente, ¿es necesario este proceso tan engorroso y confuso para atender a alumnos que están adquiriendo de forma temprana aprendizajes instrumentales, o están demostrando unas aptitudes por encima de lo esperado en comparación con sus compañeros de edad similar? Desde la perspectiva de los docentes, estos informes psicopedagógicos que les suministran no suelen ser de gran ayuda. Su cociente intelectual o los valores de unos determinados percentiles no les aporta una información útil sobre el tipo de necesidades educativas que tienen los alumnos. Lo que realmente les interesa a los docentes es saber cómo hacer que todos ellos aprendan y desarrollen sus capacidades al mayor nivel posible. Una buena convivencia entre docente y estudiante aportará una gran fuente de información, que les permitirá observar qué alumnos pueden progresar con mayor rapidez.

Ahora bien, al llegar a la universidad se produce una ruptura brusca en el seguimiento de estos estudiantes, pues desde las Consejerías de educación no pueden proporcionar a la universidad datos sobre los alumnos identificados y eso 
provoca que en la Universidad tengamos poco o nulo conocimiento en este ámbito.

El objetivo principal de este proyecto de innovación es identificar a los alumnos de altas capacidades que acceden a la universidad, en particular en el Grado en Ingeniería Informática, para experimentar y analizar diferentes metodologías de aprendizaje.

Como normalmente este tipo de alumnos no acuden al servicio de Asuntos Sociales de la Universidad el primer problema es cómo saber si un alumno ha sido identificado de altas capacidades o bien si lo es aun sin estar identificado como tal. Incluso sin llegar a alcanzar ese nivel es posible que nos encontremos alumnos de alto rendimiento a los que también deberíamos de prestar educación personalizada. En cualquiera de estos casos primero tenemos que detectarlos y luego intervenir.

El modelo de los tres anillos es un modelo que el especialista Joseph Renzulli, del Instituto de Investigación para la educación de los Alumnos Superdotados (Research Institute for Gifted Education, University of Connecticut, USA), investigó hace décadas (Renzulli J. , 1977). Consiste en tres cualidades identificables o diagnosticables que tienen que estar presentes en las personas superdotadas (Figura 1). Estas tres cualidades son: inteligencia elevada, creatividad e implicación en la tarea.

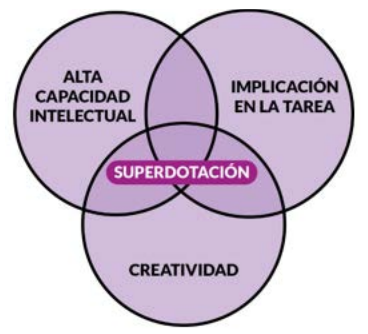

Figura 1 Modelo de los tres anillos.

Renzulli hace un especial hincapié en uno de los tres anillos, el de la inteligencia elevada, indicando que no se trata de una extraordinaria inteligencia, sino de una buena productividad o de un alto rendimiento escolar incentivado.

El anillo más fácil de identificar es el de la creatividad. Esto es debido a que la originalidad e ingenio del alumno es un rasgo más llamativo y detectable. También es cierto que pruebas como la realización de experimentos, mapas, poesías, juegos, bailes, etc., ayudan a localizar estas actitudes.

Por último, el siguiente anillo que cierra el vértice del triángulo es la implicación en las tareas. El interés y la energía que muestra el alumno superdotado a la hora de resolver unas actividades específicas le diferencia de un alumno con cociente intelectual habitual.

Estudios más recientes (Lynch, 2016) ponen de manifiesto que la detección de estos alumnos de alta capacidad es más simple y fácil de lo que parece. Los docentes deben tener presente una serie de referencias que engloban unas determinadas características generales de este tipo de alumnos, independientemente de sus condiciones particulares. Estos rasgos tan característicos están relacionados con la forma de aprender que tienen estos niños y niñas. En la tabla 1 se resumen cinco características básicas que aparecen en todos ellos, independientemente de su perfil personal o de su rendimiento escolar o académico.
Estás cinco características justifican una atención diferenciada de los más capaces con respecto al ritmo escogido por una programación didáctica estándar. No dependen de una determinada puntuación en una prueba, de un comportamiento determinado en clase o de sus calificaciones supeditadas a muchas variables diferentes a la de sus propias capacidades intelectuales. Estas cinco características nos muestran las dos vías esenciales: permitir un aprendizaje más profundo y ajustado al ritmo que demanda el alumno. Aun así, la actuación no debe ser igual para todos, cada intervención será individual atendiendo a las necesidades de cada estudiante.

Tabla 1. Características del aprendizaje de los alumnos más capaces (Lynch, 2016).

\begin{tabular}{|c|c|}
\hline Característica & Precisión \\
\hline Aprenden más rápido ... & ... sobre los temas que les interesan. \\
\hline Memorizan mejor ... & ... los datos de su preferencia. \\
\hline $\begin{array}{l}\text { Pensamiento más complejo o } \\
\text { abstracto ... }\end{array}$ & ... que sus iguales en edad. \\
\hline Pasión por temas de su interés ... & $\begin{array}{r}\text {... hasta la máxima concentración y } \\
\text { exclusión de otros temas. }\end{array}$ \\
\hline $\begin{array}{l}\text { Procesamiento paralelo de } \\
\text { estímulos ... }\end{array}$ & $\begin{array}{r}\text {... sabiendo lo que sucede a su } \\
\text { alrededor mientras se concentran en } \\
\text { una tarea especifica. }\end{array}$ \\
\hline
\end{tabular}

Las altas capacidades no son una cuestión del ser, sino más bien una cuestión del grado de desarrollo (Reyero \& Touron, 2003) (Touron, 2013). No todos los estudiantes de altas capacidades tienen un perfil determinado, por lo tanto, la actuación ha de ser adaptada a las necesidades individuales de cada persona, como hemos dicho anteriormente. En estas variables entran cuestiones como el perfil cognitivo (sus aptitudes), sus intereses, sus preferencias de aprendizaje, su personalidad y sus actitudes. A grandes rasgos la actuación deberá estar encaminada a profundizar y acelerar en mayor o menor medida, pero no todas las personas deben partir desde cero. Por ejemplo, no van a necesitar las mismas actuaciones por el mero hecho de compartir edad o fecha de detección, cada estudiante tiene su propio contexto y proceso, son diferentes entre sí. Es por esto por lo que las estrategias, las técnicas y los métodos que se llevan a cabo en el aula para atenderlos, debe ajustarse a esta diversidad sin generar un trabajo ingente que haga imposible su aplicación.

Ahora, puede que sean escasos los superdotados hallados en la aulas pero sí es más habitual encontrar alumnos de alta capacidad y/o alto rendimiento sobre los que también tenemos que intervenir. Es por esto por lo que proponemos crear aulas de desarrollo dentro de los grupos de investigación de excelencia.

\section{DESCRIPCIÓN}

Como se comentó anteriormente, las Consejerías de Educación no pueden proporcionar información sobre los alumnos de altas capacidades que llegan a la Universidad, por lo que tenemos que buscar mecanismos para identificarlos. Vemos una carencia en la formación del profesorado universitario en este tema y por ello varios miembros del equipo de trabajo hemos asistido a diferentes seminarios y jornadas sobre altas capacidades en estos últimos años pensando tanto en la detección como en la intervención y proponemos que esta formación debiera ser contemplada en el plan de formación del profesorado. 
Este estudio que presentamos lo hemos experimentado con alumnos del grado en Ingeniería Informática de la Universidad de Salamanca matriculados en diferentes cursos académicos en las asignaturas de Programación I, Programación II ambas de primer curso, Informática teórica de segundo curso y Desarrollo de Aplicaciones Avanzadas de cuarto curso.

Hemos elegido Programación I y II para tener la certeza de que llegamos a todos los alumnos que ingresan en el grado y que les podemos observar a lo largo de todo el curso académico. Al ser asignaturas con una gran carga práctica permiten que el alumno participe en el aula, demuestre su creatividad y se implique en la realización de las tareas.

Así, con los conocimientos adquiridos y aplicando la teoría de Lunch, al finalizar Programación I hemos detectado a potenciales alumnos de altas capacidades, alguno de ellos posible superdotado, y basándonos en los resultados de evaluación a aquellos que consideramos posibles de alto rendimiento. Es en este momento cuando iniciamos la labor de intervención, manteniendo tutorías individualizadas, dándoles a conocer el grupo de investigación en bioinformática, sistemas informáticos inteligentes y tecnología educativa (BISITE, 2021) y presentándoles las diferentes líneas de investigación en las que trabaja el grupo para ofrecerles la posibilidad de desarrollar su capacidad a su ritmo particular y en diferentes temáticas en función de sus intereses.

Podríamos crear un laboratorio de desarrollo por cada una de las líneas de investigación, que en este caso son múltiples, en concreto: inteligencia artificial, machine learning, sistemas emocionales, Fintech, IoT, blockchain, industria 4.0, smart cities, smart grids, textiles inteligentes, etc. En esta fase, como son los estudiantes los que deciden en qué línea quieren integrarse, sólo hemos desarrollado los laboratorios de aplicaciones móviles de nueva generación, IoT y blockchain.

En cada laboratorio siempre hay un profesor del grupo de investigación responsable del seguimiento de los estudiantes. La propuesta es que ese profesor también haga una labor de orientador académico para evitar el fracaso, ya que la estadística marca que las altas capacidades no siempre están asociadas con el alto rendimiento y así lo hemos experimentado en los laboratorios de desarrollo. Además, pretendemos que estos alumnos puedan ser mentores de otros compañeros no iniciados en este proyecto, pues en muchas ocasiones entre ellos es más fácil el entendimiento y comprensión.

Desde estos laboratorios de desarrollo proponemos llevar a cabo un programa de divulgación de la investigación desarrollada que al menos implique la realización de charlas, conferencias, seminarios y, por qué no, pequeñas publicaciones en las que participen activamente los alumnos de altas capacidades o alto rendimiento y que puedan tener un reconocimiento académico. Con esta iniciativa conseguiríamos el desarrollo de los alumnos que están participando en el programa y a la vez sería una forma de abrir la puerta a esos alumnos de altas capacidades que aún no han llegado a la universidad pero que pueden estar participando en otros programas, como por ejemplo el Bachillerato de excelencia de la Junta de Castilla y León, dando así continuidad a esos programas.

Ahora bien, no es fácil detectar a los alumnos que tienen este tipo de necesidad especial, entre otras cosas por la falta de formación del profesorado universitario en este campo, Por ello en este proyecto proponemos seguir observando a los alumnos a lo largo del grado, realizando de nuevo la detección en otras asignaturas de los cursos superiores para así poder ofrecerles su inclusión en el proyecto a los no detectados en primero. También en esas mismas asignaturas de los cursos posteriores podemos hacer un seguimiento del rendimiento académico de los alumnos que participan en los laboratorios de desarrollo, analizando el interés de las actividades realizadas y valorando los beneficios que les proporcionan.

\section{Resultados}

A fecha de junio de 2021 en el grupo de investigación BISITE hay 9 alumnos en los laboratorios de desarrollo distribuidos entre las líneas de aplicaciones móviles, IoT y blockchain. La política del grupo para responder a las necesidades especiales de estos alumnos e incentivar su participación ha sido concederles una beca de estudios soportada por el propio grupo de investigación, por lo que el acceso es muy restringido; los primeros años se admitió solo un estudiante por año, sin embargo, en el curso académico 201920 y 2020-21 hemos podido admitir a varios.

Para medir el impacto de la creación de estos laboratorios de desarrollo hemos recabado la opinión de los propios estudiantes que participan en esta iniciativa mediante un formulario anónimo del que pasamos a exponer los resultados.

Una de las preguntas es ¿Te identificas como alumno de alguno de estos tipos? (Figura 2) Como se refleja tres alumnos son de altas capacidades y no alto rendimiento, otros dos se identifican como de alto rendimiento y los otros cuatro no se identifican en ninguna de las dos categorías, por lo que entendemos que ellos mismos asocian sus buenos resultados académicos al esfuerzo constante y no a pertenecer a uno de esos perfiles. Sin duda este resultado pone de manifiesto que debemos de mejorar la forma de detectar alumnos de altas capacidades y/o alto rendimiento.

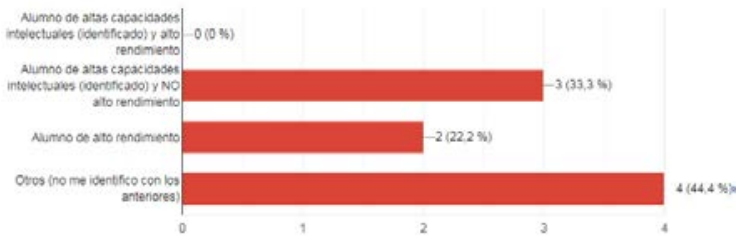

Figura 2. ¿Te identificas como alumno de alguno de estos tipos?

Esta iniciativa la llevamos desarrollando varios cursos académicos y les hemos preguntado por el tiempo que llevan en el proyecto, pues así se pone de manifiesto su satisfacción pues lo empezamos a experimentar con un solo alumno en el curso académico 2017-18 y se mantienen en el proyecto (Figura 3).
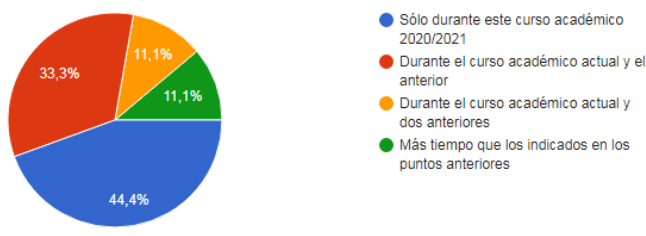

Figura 3. ¿Cuántos cursos académicos llevas participando en el laboratorio de desarrollo?

A los estudiantes les preguntamos sobre si consideran positiva esta experiencia y si la deberíamos llevar a otros grupos 
de investigación y claramente la respuesta es afirmativa (Figura 4).

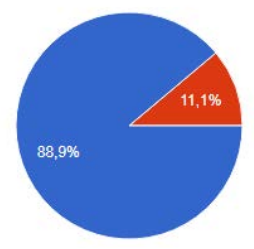

- Sí

Figura 4. ¿Consideras que la experiencia es positiva y habría que extenderla a otros grupos de investigación?

También a los alumnos de los laboratorios de desarrollo les preguntamos (Figura 5) si les gustaría ser mentores de otros alumnos de cursos inferiores y si consideran adecuado que la Universidad reconociera de alguna manera esta labor, por ejemplo, con ayudas para asistir a jornadas, congresos, seminarios, etc.

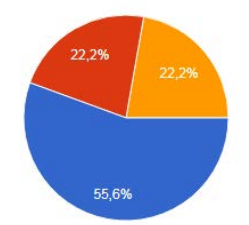

$\because$ : si

No me sientio capaz

Figura 5. ¿Te gustaría ser mentor de otros alumnos de cursos inferiores?

Por último, hay que destacar la respuesta que dieron a la pregunta sobre si les hubiera gustado participar en los laboratorios de desarrollo durante los estudios de bachillerato, pensando poder ofrecer dicha participación a estudiantes preuniversitarios identificados de altas capacidades (Figura 6).

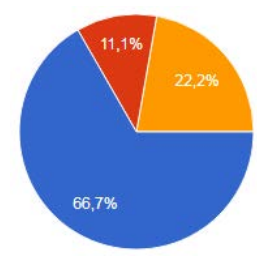

$$
\text { - Sí }
$$$$
\text { No lo tengo claro }
$$

Figura 6. ¿Crees que los laboratorios de desarrollo se deberían de ofrecer para los alumnos de Bachillerato?

\section{CONCLUSIONES}

Hay que dar continuidad en la atención a los alumnos de altas capacidades $\mathrm{y} / \mathrm{o}$ alto rendimiento en todos los niveles educativos, en particular durante su formación universitaria.

Facilitar el acceso a la universidad a alumnos identificados de altas capacidades, así como a los de alto rendimiento que participen en diferentes programas, como el bachillerato de excelencia o aquellos que finalizan los estudios en un conservatorio de nivel profesional.

Establecer un mecanismo que permita identificar a alumnos de altas capacidades en las universidades y alto rendimiento e intervenir mediante laboratorios de desarrollo dentro de los grupos de investigación de excelencia, a ser posible becados por el Ministerio de Educación y Formación Profesional para que todos los grupos tengan la posibilidad de desarrollar estos laboratorios.

Motivar a los alumnos que participan en estos laboratorios para ser mentores de sus compañeros de estudios mediante un reconocimiento académico y otro tipo de incentivo.

\section{AGRADECIMIENTOS}

A la Universidad de Salamanca por la convocatoria de ayudas a proyectos de innovación y mejora docente por la concesión de la propuesta Innovando en educación superior con alumnado de altas capacidades (Código del Proyecto: ID2020/097) que nos ha permitido desarrollar este estudio.

Sin duda, agradecer al grupo de investigación de la Universidad de Salamanca, BISITE (Bioinformática, Sistemas Informáticos Inteligentes y Tecnología Educativa) en el que se ha puesto en marcha la iniciativa presentada en este artículo.

\section{REFERENCIAS}

BISITE. (10 de 5 de 2021). Grupo de investigación en Bioinformática, Sistemas Informáticos Inteligentes $y$ Tecnología Educativa. Obtenido de bisite.usal.es

BOE. (2014). Real Decreto 412/2014, de 6 de junio, por el que se establece la normativa básica de los procedimientos de admisión a las enseñanzas universitarias oficiales de Grado.

Educacyl., P. d.-1. (25 de 5 de 2021). Dirección provincial de Valladolid. Obtenido de https://www.educa.jcyl.es/dpvalladolid/es/informacionespecifica-dp-valladolid/area-programaseducativos/atencion-diversidad-equidadeducativa/atencion-alumnado-altas-capacidadesintelectuales

Fernández-Molina, M. (2021). Mentoría y altas capacidades intelectuales. Modelos y experiencias prácticas. Ediciones Pirámide.

Jiménez, C. (2000). Diagnóstico y educación de los más capaces. Madrid: UNED-Comunidad de Madrid-MEC.

Lynch, M. (2016). 5 Ways Gifted Students Learn Differently. https://www.theedadvocate.org/5-ways-gifted-studentslearn-differently/ Último acceso 16/06/2021.

Renzulli, J. (1977). The Enrichment Triad Model: A guide for developing defensible programs for the gifted and talented. Mansfield Center, CT: Creative Learning Press.

Renzulli, J., \& Reis, S. (1997). The Schoolwide Enrichment Model: A How-To Guide for Educational Excellence. Creative Learning Press. Connecticut.

Reyero, M., \& Touron, J. (2003). REYERO, Marta y TOURON, Javier; El desarrollo del talento; la aceleración como estrategia educativa. Netbiblo, A Coruña.. A Coruña: Netbiblo.

The mentorac project-UMA, M. (s.f.). Obtenido de https://mentorac.es/

Tomlinson, C. (2013). El aula diversificada. Barcelona: Ediciones Octaedro.

Touron, J. (2013). 11 razones por las que los alumnos necesitan una atención educativa diferenciada (3/6. https://www.javiertouron.es/11-razones-por-las-que-losalumnos/ Último acceso el 12/06/2021. 